TIFR/TH/04-13

June 2004

\title{
Looking for the Charged Higgs Boson
}

\author{
D. P. Roy \\ Department of Theoretical Physics \\ Tata Institute of Fundamental Research \\ Homi Bhabha Road, Mumbai 400 005, India \\ dproy@theory.tifr.res.in
}

\begin{abstract}
This review article starts with a brief introduction to the charged Higgs boson $\left(H^{ \pm}\right)$in the Minimal Supersymmetric Standard Model (MSSM). It then discusses the prospects of a relatively light $H^{ \pm}$boson search via top quark decay at Tevatron/LHC, and finally a heavy $H^{ \pm}$boson search at LHC. The viable channels for $H^{ \pm}$search are identified in both the cases, with particular emphasis on the $H^{ \pm} \rightarrow \tau \nu$ decay channel. The effects of NLO QCD correction in the SM as well as the MSSM are discussed briefly.
\end{abstract}

\section{Introduction}

The minimal supersymmetric extension of the Standard Model (MSSM) contains two Higgs doublets $\phi_{u}^{+, 0}$ and $\phi_{d}^{0,-}$, with opposite hypercharge $Y= \pm 1$, to give masses to the up and down type quarks and leptons. This also ensures anomaly cancellation between their fermionic partners. The two doublets of complex scalars correspond to 8 degrees of freedom, 3 of which are absorbed as Goldstone bosons to give mass and longitudinal components to the $W^{ \pm}$and $Z$ bosons. This leaves 5 physical states: two neutral scalars $h^{0}$ and $H^{0}$, a pseudoscalar $A^{0}$, and a pair of charged Higgs bosons $H^{ \pm}$. While it may be hard to distinguish any one of these neutral Higgs bosons from that of the Standard Model, the $H^{ \pm}$pair carry a 
distinctive hall-mark of the MSSM. Hence the charged Higgs boson plays a very important role in the search of the SUSY Higgs sector.

\section{$1.1 \quad$ Masses and Couplings}

At the tree-level all the MSSM Higgs masses and couplings are given in terms of two parameters - the ratio of the vacuum expectation values, $\tan \beta=\left\langle\phi_{u}^{0}\right\rangle /\left\langle\phi_{d}^{0}\right\rangle$, and any one of the masses, usually taken to be $M_{A}$. The physical $H^{ \pm}$and $A^{0}$ states correspond to the combinations

$$
\begin{aligned}
H^{ \pm} & =\phi_{u}^{ \pm} \cos \beta+\phi_{d}^{ \pm} \sin \beta, \\
A^{0} & =\sqrt{2}\left(\operatorname{Im} \phi_{u}^{0} \cos \beta+\operatorname{Im} \phi_{d}^{0} \sin \beta\right),
\end{aligned}
$$

while their masses are related by

$$
M_{H^{ \pm}}^{2}=M_{A}^{2}+M_{W}^{2}
$$

with negligible radiative corrections [1]. However, the neutral scalars get a large radiative correction from the top quark loop along with the top squark loop,

$$
\epsilon=\frac{3 g^{2} m_{t}^{4}}{8 \pi^{2} M_{W}^{2}} \ln \left(\frac{M_{\tilde{t}}^{2}}{m_{t}^{2}}\right)
$$

where $M_{\tilde{t}}$ denotes the average mass of the two top squarks $\left(\tilde{t}_{1,2}\right)$. Including this radiative correction, the mass-squared matrix of the neutral scalars is given by

$$
\left(\begin{array}{cc}
M_{A}^{2} \sin ^{2} \beta+M_{Z}^{2} \cos ^{2} \beta & -\left(M_{A}^{2}+M_{Z}^{2}\right) \sin \beta \cos \beta \\
-\left(M_{A}^{2}+M_{Z}^{2}\right) \sin \beta \cos \beta & M_{A}^{2} \cos ^{2} \beta+M_{Z}^{2} \sin ^{2} \beta+\epsilon^{\prime}
\end{array}\right),
$$

where $\epsilon^{\prime}=\epsilon / \sin ^{2} \beta$. Thus the physical $h^{0}$ and $H^{0}$ masses correspond to the eigen values

$M_{h, H}^{2}=\frac{1}{2}\left[M_{A}^{2}+M_{Z}^{2}+\epsilon^{\prime} \mp\left\{\left(M_{A}^{2}+M_{Z}^{2}+\epsilon^{\prime}\right)^{2}-4 M_{A}^{2} M_{Z}^{2} \cos ^{2} \beta-4 \epsilon^{\prime}\left(M_{A}^{2} \sin ^{2} \beta+M_{Z}^{2} \cos ^{2} \beta\right)\right\}^{1 / 2}\right]$.

The corresponding eigen vectors are the two orthogonal combinations of $\operatorname{Re} \phi_{u, d}^{0}$ with mixing angle $\alpha$, which diagonalizes this matrix, i.e.

$$
\tan 2 \alpha=\tan 2 \beta \frac{M_{A}^{2}+M_{Z}^{2}}{M_{A}^{2}-M_{Z}^{2}+\epsilon^{\prime} / \cos 2 \beta},-\pi / 2<\alpha<0 .
$$

For $M_{A} \gg M_{Z}, \alpha \rightarrow \beta-\pi / 2$. Note that $M_{H}^{2}$ and $M_{H^{ \pm}}^{2} \rightarrow M_{A}^{2}$, while the lighter scalar mass approaches a finite limit

$$
M_{h}^{2} \stackrel{M_{A} \gg M_{Z}}{\longrightarrow} M_{Z}^{2} \cos ^{2} 2 \beta+\epsilon .
$$

Finally there is an additional radiative contribution to this limit from $\tilde{t}_{L, R}$ mixing $[1,2]$,

$$
\epsilon_{\mathrm{mix}}=\frac{3 g^{2} m_{t}^{4}}{8 \pi^{2} M_{W}^{2}} \frac{X_{t}^{2}}{M_{\tilde{t}}^{2}}\left(1-\frac{X_{t}^{2}}{12 M_{\tilde{t}}^{2}}\right) \leq \frac{9 g^{2} m_{t}^{4}}{8 \pi^{2} M_{W}^{2}}
$$


Table 1: Important couplings of the MSSM neutral Higgs bosons $h, H$ and $A$ relative to those of the SM Higgs boson.

\begin{tabular}{|c|c|c|c|c|}
\hline Channel & $H_{\mathrm{SM}}$ & $h$ & $H$ & $A$ \\
\hline $\bar{b} b\left(\tau^{+} \tau^{-}\right)$ & $\frac{g m_{b}}{2 M_{W}}\left(m_{\tau}\right)$ & $-\sin \alpha / \cos \beta$ & $\cos \alpha / \cos \beta$ & $\tan \beta$ \\
& $\rightarrow 1$ & $\tan \beta$ & $"$ \\
\hline $\bar{t} t$ & $g \frac{m_{t}}{2 M_{W}}$ & $\cos \alpha / \sin \beta$ & $\sin \alpha / \sin \beta$ & $\cot \beta$ \\
& & $\rightarrow 1$ & $\cot \beta$ & $"$ \\
\hline$W W(Z Z)$ & $g M_{W}\left(M_{Z}\right)$ & $\sin (\beta-\alpha)$ & $\cos (\beta-\alpha)$ & 0 \\
& & $\rightarrow 1$ & 0 & \\
\hline
\end{tabular}

where $X_{t}=A_{t}-\mu \cot \beta$. Thus while $\epsilon_{\text {mix }}$ is a function of the SUSY breaking trilinear coupling $A_{t}$ and the Higgsino mass parameter $\mu$, it has a constant upper limit $\left(\sim M_{W}^{2}\right)$, which is reached at $X_{t}^{2}=6 M_{\tilde{t}}^{2}$. One can also check from (3) that $\epsilon \sim M_{W}^{2}$ for a SUSY breaking scale of $M_{\tilde{t}} \sim 1 \mathrm{TeV}$. Adding the nonleading radiative contributions to eqs.(7) and (8) gives a limit on the light scalar mass

$$
M_{h} \stackrel{M_{A} \gg M_{Z}}{\longrightarrow} 118 \mathrm{GeV}(130 \mathrm{GeV}) \text { at } \tan \beta=3(30)
$$

for the top quark pole mass of $175 \mathrm{GeV}[1,2]$.

Table 1 shows the important couplings of the MSSM neutral Higgs bosons relative to those of the SM Higgs boson. The limiting values of these couplings at large $M_{A}$ are indicated by arrows. The important couplings of the charged Higgs boson, which has no SM analogue, are

$$
\begin{aligned}
H^{+} \bar{t} b & : \frac{g}{\sqrt{2} M_{W}}\left(m_{t} \cot \beta+m_{b} \tan \beta\right), H^{+} \tau \nu: \frac{g}{\sqrt{2} M_{W}} m_{\tau} \tan \beta, \\
H^{+} \bar{c} s & : \frac{g}{\sqrt{2} M_{W}}\left(m_{c} \cot \beta+m_{s} \tan \beta\right), H^{+} W^{-} Z: 0,
\end{aligned}
$$

with negligible radiative corrections.

The coefficients of the fermion mass terms of eq.(10) and Table 1 reflect the compositions of the respective Higgs bosons in terms of $\phi_{u, d}$. It is clear from eqs.(2) and (10) that measurements of $H^{ \pm}$mass and couplings will determine the masses and couplings of the other MSSM Higgs bosons via the underlying parameters $M_{A}$ and $\tan \beta$. 


\subsection{Indirect constraints on $\tan \beta$ and $M_{A}$}

The $H^{+} \bar{t} b$ Yukawa coupling of eq.(10) is ultraviolet divergent. Assuming it to remain perturbative upto the GUT scale implies

$$
1<\tan \beta<m_{t} / m_{b}(\sim 50) .
$$

However this assumes the absence of any new physics beyond the MSSM upto the GUT scale - i.e. the socalled desert scenario. Without this assumption one gets weaker limits from the perturbative bounds on this coupling at the electroweak scale, i.e.

$$
0.3<\tan \beta<200 .
$$

Moreover there is a strong constraint on the $M_{A}-\tan \beta$ parameter space coming from the LEP-2 bound on the $H_{S M}$ mass, which is also applicable to $M_{h}$ at low $\tan \beta$, i.e. $M_{h}>114$ $\mathrm{GeV}$ [2]. Comparing this with the MSSM prediction (9) implies $\tan \beta>2.4$ for any value of $M_{A}$ [1, 2] (see Fig. 4 below). Note however from eqs. (3),(7) and (8) that the MSSM prediction depends sensitively on the top quark mass. The recent increase of this mass from 175 to $178 \pm 4.3 \mathrm{GeV}$ [3] along with a more exact evaluation of the radiative correction [4] have resulted in a significant weaking of this constraint. In fact there is no LEP bound on $\tan \beta$ now, which would be valid for all values of $M_{A}$. Nonetheless it implies $M_{A}>150 \mathrm{GeV}$ $\left(M_{H^{ \pm}}>170 \mathrm{GeV}\right)$ over the low $\tan \beta(\leq 2)$ region. But being an indirect bound, it depends strongly on the underlying model. There is no such bound in the CP violating MSSM due to $h$ - $A$ mixing [5]. Moreover there are singlet extensions of the MSSM Higgs sector like the socalled NMSSM, which invalidate these $M_{A}\left(M_{H^{ \pm}}\right)$bounds without disturbing the charged Higgs boson [6]. In fact there is an additional contribution to the tree-level mass relation (2) in the NMSSM, which permits $H^{ \pm}$to be even lighter than the $W$ boson. Therefore it is prudent to relax these indirect constraints on $M_{H^{ \pm}}$and $\tan \beta$, and search for $H^{ \pm}$over the widest possible parameter space. It should be noted here that the $H^{ \pm}$couplings of eq.(10) continue to hold over a wide class of models. In fact the fermionic couplings hold for the general class of Type-II two-Higgs-doublet models, where one doublet couples to up type and the other to down type quarks and leptons [1].

\subsection{Direct $H^{ \pm}$Mass Limit from LEP}

Figure 1 shows a direct mass limit of $M_{H^{ \pm}}>80 \mathrm{GeV}$ from LEP-2, which is in agreement with the MSSM prediction (2). It is based on both the decay channels $H^{ \pm} \rightarrow c s$ and $\tau \nu$ of eq.(10). Hence it is a robust limit, spanning the full $\tan \beta$ range of eq.(12). The limit is broadly restricted to the $W$ mass region because of the $W^{+} W^{-}$background. However one gets a slightly stronger limit $(>90 \mathrm{GeV})$ from the $\tau \nu$ channel, which is reflected in the $\tan \beta>1$ region.

\section{Search for a Light $H^{ \pm}\left(M_{H^{ \pm}}<m_{t}\right)$ at Tevatron/LHC}

The main production mechanism in this case is top quark pair production

$$
q \bar{q}, g g \rightarrow t \bar{t},
$$


followed by

$$
t \rightarrow b H^{+} \text {and/or } \bar{t} \rightarrow \bar{b} H^{-} .
$$

The dominant decay channels of $H^{ \pm}$are

$$
H^{+} \rightarrow c \bar{s}, \tau^{+} \nu \text { and } W b \bar{b}+h c,
$$

where the 3-body final state comes via the virtual $t \bar{b}$ channel. All these decay widths are easily calculated from the Yukawa couplings of eq.(10). The QCD correction can be simply implemented in the leading log approximation by substituting the quark masses appearing in the Yukawa couplings by their running masses at the $H^{ \pm}$mass scale [7]. Its main effect is to reduce the $b$ and $c$ pole masses of 4.6 and $1.8 \mathrm{GeV}$ respectively [2] to their running masses $m_{b}\left(M_{H^{ \pm}}\right) \simeq 2.8 \mathrm{GeV}$ and $m_{c}\left(M_{H^{ \pm}}\right) \simeq 1 \mathrm{GeV}$. The corresponding reduction in the $t$ pole mass of $175 \mathrm{GeV}$ is only $\sim 5 \%$.

The resulting branching ratios for the four decay processes of (14) and (15) are shown in Fig. 2 against $\tan \beta$ for a representative $H^{ \pm}$mass of $140 \mathrm{GeV}$. The $t \rightarrow b H^{+}$branching ratio is seen to be large at $\tan \beta \lesssim 1$ and $\tan \beta \gtrsim m_{t} / m_{b}$, which are driven by the $m_{t}$ and the $m_{b}$ terms of the $H^{+} \bar{t} b$ coupling respectively. However it has a pronounced minimum around $\tan \beta=\sqrt{m_{t} / m_{b}} \simeq 7.5$, where the SM decay of $t \rightarrow b W$ is dominant. The $H^{ \pm}$is expected to decay dominantly into the $\tau \nu$ channel for $\tan \beta>1$, while the $c s$ and the $b \bar{b} W$ channels dominate in the $\tan \beta \leq 1$ region. This can be easily understood in terms of the respective couplings of eq.(10). Note however that the $H^{+} \rightarrow \bar{b} b W$ three-body decay via virtual $t \bar{b}$ channel is larger than the $H^{+} \rightarrow c \bar{s}$ decay for $M_{H^{ \pm}} \gtrsim 140 \mathrm{GeV}$, although the former is a higher order process $[8,9]$. This is because the $H^{+} \bar{t} b$ coupling is larger than the $H^{+} \bar{c} s$ coupling by a factor of $m_{t} / m_{c}>100$ in the low $\tan \beta$ region.

\section{1 $H^{ \pm}$Search in the $c s$ and $b \bar{b} W$ Channels $(\tan \beta<1)$}

One can look for a possible top quark decay into the $H^{ \pm} \rightarrow c s$ channel in the Tevatron $t \bar{t}$ data in the leptonic and dileptonic channels using the so called indirect or disappearance method [10]. Here

$$
\sigma_{t \bar{t}}^{\ell}=\sigma_{t \dot{\bar{t}}} 2 B_{\ell}\left(1-B_{\ell}\right), \sigma_{t \bar{t}}^{\ell \ell}=\sigma_{t \dot{\bar{t}}} B_{\ell}^{2}
$$

Using the QCD prediction for $\sigma_{t \bar{t}}=5-5.5 p b[11]$ and SM prediction for $B_{\ell} \equiv B(t \rightarrow$ $(e, \mu) \nu b)=2 / 9$ one can predict the number of such events. In the presence of $t \rightarrow b H(H \rightarrow$ $c s$ ) decay channel one expect a reduction in $B_{\ell}$ and hence the number of $t \bar{t}$ events in the leptonic and dileptonic channels with respect to the SM prediction.

No such reduction was found in the $t \bar{t}$ data of the $D \emptyset[12]$ and CDF [13] experiments. The resulting exclusion region in the $M_{H^{ \pm}}-\tan \beta$ parameter space is shown on the left side of Fig. 1. It is seen to exclude the $M_{H^{ \pm}}<130 \mathrm{GeV}, \tan \beta<1$ region, where the $H^{ \pm} \rightarrow c s$ is the dominant decay mode. Indeed a comparison with Fig. 2 shows that the bulk of the parameter space for which $H^{ \pm} \rightarrow c s$ is the dominant decay mode is already excluded by these data. This method is no longer applicable for $M_{H^{ \pm}} \geq 140 \mathrm{GeV}$, where $H^{ \pm} \rightarrow b \bar{b} W$ is the dominant decay mode. Here the signal consists of $t \rightarrow b \bar{b} b W$ events against the SM background of $t \rightarrow b W$, followed by either leptonic or hadronic decay of $W$. So one has to look for an excess of $b$ tags in the $t \bar{t}$ events compared to the SM prediction [9]. With a large 
number of $t \bar{t}$ events expected from the future Tevatron Runs and especially from the LHC one expects to use this method to extend the $H^{ \pm}$probe to significantly higher values of $M_{H^{ \pm}}$ at low $\tan \beta(\lesssim 1)$.

\section{2 $\quad H^{ \pm}$Search in the $\tau \nu$ Channel $(\tan \beta>1)$}

As discussed earlier the $\tan \beta>1$ region is theoretically favoured. Fig. 2 shows that $H^{ \pm} \rightarrow \tau \nu$ is the dominant decay mode over this region. Therefore the $\tau$ channel is the most important channel for $H^{ \pm}$search. The above mentioned disappearance (indirect) method is equally applicable to this channel. The resulting exclusion regions from the $D \emptyset[12]$ and CDF [13] experiments can be seen on the right side of Fig. 1. Evidently the disappearance method is not viable when the signal is $\lesssim 10 \%$ of the SM background, since this is the typical uncertainty in the QCD prediction of $\sigma_{t \bar{t}}$. This explains why the resulting exclusion regions cover only extreme values of $\tan \beta$ (compare Fig. 2). In order to extend the probe to the theoretically favoured range of $\tan \beta=1-50$, one has to directly search for the $t \rightarrow b \tau \nu$ events. Using the universality of $W$ coupling one can easily predict the number of $t \rightarrow b \tau \nu$ events via $W$ from that of $t \rightarrow b \ell \nu$ events. Since $H^{ \pm}$couples only to the former any excess of $\tau$ events over the universality prediction constitutes a signal for $t \rightarrow b H^{ \pm}$decay. The CDF group has used a small data sample in the inclusive $\tau$ channel to search for the direct $t \rightarrow b H^{ \pm}$signal [14]. The resulting exclusion region can be seen on the right side of Fig. 1, which is roughly overlapping with that obtained via the indirect method. With the much higher event rates expected from future Tevatron Runs and LHC it will be better to use the $\ell \tau$ channel for $H^{ \pm}$search instead of the inclusive $\tau$, since the former is a cleaner and far more robust channel $[15,16]$. It corresponds to the decay of one of the $t \bar{t}$ pair into $\ell$ via $W$ while the other decays into a $\tau$ channel.

\section{$2.3 \tau$ Polarization Effect}

The discovery reach of the $\tau$ channel for $H^{ \pm}$search at Tevatron and LHC can be significantly enhanced by exploiting the opposite polarization of $\tau$ coming from the $H^{ \pm} \rightarrow \tau \nu\left(P_{\tau}=\right.$ $+1)$ and $W^{ \pm} \rightarrow \tau \nu\left(P_{\tau}=-1\right)$ decays [17]. Let us briefly describe this simple but very powerful method. The best channel for $\tau$-detection in terms of efficiency and purity is its 1-prong hadronic decay channel, which accounts for $50 \%$ of its total decay width. The main contributors to this channel are

$$
\begin{aligned}
& \tau^{ \pm} \rightarrow \pi^{ \pm} \nu_{\tau}(12.5 \%), \quad \tau^{ \pm} \rightarrow \rho^{ \pm} \nu_{\tau} \rightarrow \pi^{ \pm} \pi^{0} \nu_{\tau}(26 \%), \\
& \tau^{ \pm} \rightarrow a_{1}^{ \pm} \nu_{\tau} \rightarrow \pi^{ \pm} \pi^{0} \pi^{0} \nu_{\tau}(7.5 \%),
\end{aligned}
$$

where the branching fractions of the $\pi$ and $\rho$ channels include the small $K$ and $K^{*}$ contributions respectively [2], which have identical polarization effects. Together they account for more than $90 \%$ of the 1-prong hadronic decay of $\tau$. The CM angular distributions of $\tau$ decay into $\pi$ or a vector meson $v\left(=\rho, a_{1}\right)$ is simply given in terms of its polarization as

$$
\frac{1}{\Gamma_{\pi}} \frac{d \Gamma_{\pi}}{d \cos \theta}=\frac{1}{2}\left(1+P_{\tau} \cos \theta\right)
$$




$$
\begin{aligned}
\frac{1}{\Gamma_{v}} \frac{d \Gamma_{v L}}{d \cos \theta} & =\frac{\frac{1}{2} m_{\tau}^{2}}{m_{\tau}^{2}+2 m_{v}^{2}}\left(1+P_{\tau} \cos \theta\right), \\
\frac{1}{\Gamma_{v}} \frac{d \Gamma_{v T}}{d \cos \theta} & =\frac{m_{v}^{2}}{m_{\tau}^{2}+2 m_{v}^{2}}\left(1-P_{\tau} \cos \theta\right),
\end{aligned}
$$

where $L, T$ denote the longitudinal and transverse polarization states of the vector meson $[17,18]$. This angle is related to the fraction $x$ of the $\tau$ lab. momentum carried by the meson, i.e. the (visible) $\tau$-jet momentum, via

$$
\cos \theta=\frac{2 x-1-m_{\pi, v}^{2} / m_{\tau}^{2}}{1-m_{\pi, v}^{2} / m_{\tau}^{2}} .
$$

It is clear from (18) and (19) that the signal $\left(P_{\tau}=+1\right)$ has a harder $\tau$-jet than the background $\left(P_{\tau}=-1\right)$ for the $\pi$ and the $\rho_{L}, a_{1 L}$ contributions; but it is the opposite for $\rho_{T}, a_{1 T}$ contributions. Now, it is possible to suppress the transverse $\rho$ and $a_{1}$ contributions and enhance the hardness of the signal $\tau$-jet relative to the background even without identifying the individual resonance contributions to this channel. This is because the transverse $\rho$ and $a_{1}$ decays favour even sharing of momentum among the decay pions, while the longitudinal $\rho$ and $a_{1}$ decays favour uneven distributions, where the charged pion carries either very little or most of the momentum $[17,18]$. Figure 3 shows the decay distributions of $\rho_{L}, a_{1 L}$ and $\rho_{T}, a_{1 T}$ in the momentum fraction carried by the charged pion, i.e.

$$
x^{\prime}=p_{\pi \pm} / p_{\tau-\mathrm{jet}} .
$$

The distributions are clearly peaked near $x^{\prime} \simeq 0$ and $x^{\prime} \simeq 1$ for the longitudinal $\rho$ and $a_{1}$, while they are peaked in the middle for the transverse ones. Note that the $\tau^{+} \rightarrow \pi^{ \pm} \nu_{\tau}$ decay would appear as a $\delta$ function at $x^{\prime}=1$ on this plot. Thus requiring the $\pi^{ \pm}$to carry $>80 \%$ of the $\tau$-jet momentum,

$$
x^{\prime}>0.8,
$$

retains about half the longitudinal $\rho$ along with the pion but very little of the transverse contributions. This cut suppresses not only the $W \rightarrow \tau \nu$ background but also the fake $\tau$ background from QCD jets ${ }^{1}$. Consequently the $\tau$-channel can be used for $H^{ \pm}$search over a wider range of parameters. The resulting $H^{ \pm}$discovery reach of LHC is shown on the left side of Fig.4 [19]. It goes upto $M_{A} \simeq 100 \mathrm{GeV}\left(M_{H^{ \pm}} \simeq 130 \mathrm{GeV}\right)$ around the dip region of $\tan \beta \simeq 7.5$ and upto $M_{A} \simeq 140 \mathrm{GeV}\left(M_{H^{ \pm}} \simeq 160 \mathrm{GeV}\right)$ outside this region.

\section{Search for a Heavy $H^{ \pm}\left(M_{H^{ \pm}}>m_{t}\right)$ at LHC}

The main production process here is the leading order (LO) process [20]

$$
g b \rightarrow t H^{-}+\text {h.c. }
$$

The complete NLO QCD corrections have been recently calculated by two groups [21, 22], in agreement with one another. Their main results are summarized below:

\footnotetext{
${ }^{1}$ Note that the $x^{\prime} \simeq 0$ peak from $\rho_{L}$ and $a_{1 L}$ can not be used in practice, since $\tau$-identification requires a hard $\pi^{ \pm}$, which will not be swept away from the accompanying neutrals by the magnetic field.
} 
(i) The effect of NLO corrections can be incorporated by multiplying the above LO crosssection by a $K$ factor, with practically no change in its kinematic distributions.

(ii) With the usual choice of renormalization and factorization scales, $\mu_{R}=\mu_{F}=M_{H^{ \pm}}+$ $m_{t}$, one gets $K \simeq 1.5$ over the large $M_{H^{ \pm}}$and $\tan \beta$ range of interest.

(iii) The overall NLO correction of $50 \%$ comes from two main sources - (a) 80\% correction from gluon emission and virtual gluon exchange contributions to the LO process (22), and (b) $\sim-30 \%$ correction from the NLO process

$$
g g \rightarrow t H^{-} b+h . c .
$$

after subtracting the overlapping piece from (22) to avoid double counting.

(iv) As clearly shown in [22], the negative correction from (b) is an artifact of the common choice of factorization and renormalization scales. With a more appropriate choice of the factorization scale, $\mu_{F} \simeq\left(M_{H^{ \pm}}+m_{t}\right) / 5$, the correction from (b) practically vanishes while that from (a) reduces to $\sim 60 \%$. Note however that the overall $K$ factor is insensitive to this scale variation.

(v) Hence for simplicity one can keep a common scale of $\mu_{F, R}=M_{H^{ \pm}}+m_{t}$ along with a $K$ factor of 1.5 , with an estimated uncertainty of $20 \%$. Note that for the process $(22)$ the running quark masses of the $H^{+} \bar{t} b$ coupling (10) are to be evaluated at $\mu_{R}$, while the patron densities are evaluated at $\mu_{F}$.

The dominant decay mode for a heavy $H^{ \pm}$is into the $t b$ channel. The $H^{ \pm} \rightarrow \tau \nu$ is the largest subdominant channel at large $\tan \beta(\gtrsim 10)$, while the $H^{ \pm} \rightarrow W^{ \pm} h^{0}$ can be the largest subdominant channel over a part of the small $\tan \beta$ region [1]. Let us look at the prospects of a heavy $H^{ \pm}$search at LHC in each of these channels. The dominant background in each case comes from the $t \bar{t}$ production process (13).

\subsection{Heavy $H^{ \pm}$Search in the $\tau \nu$ Channel}

This constitutes the most important channel for a heavy $H^{ \pm}$search at LHC in the large $\tan \beta$ region. Over a large part of this region, $\tan \beta \gtrsim 10$ and $M_{H^{ \pm}} \gtrsim 300 \mathrm{GeV}$, we have

$$
B R\left(H^{ \pm} \rightarrow \tau \nu\right)=20 \pm 5 \%
$$

The $H^{ \pm}$signal coming from (22) and (24) is distinguished by very hard $\tau$-jet and missing$p_{T}\left(p_{T}\right)$,

$$
p_{\tau-\mathrm{jet}}^{T}>100 \mathrm{GeV} \text { and } \not p_{T}>100 \mathrm{GeV},
$$

with hadronic decay of the accompanying top quark $(t \rightarrow b q \bar{q})$ [23]. The main background comes from the $t \bar{t}$ production process (13), followed by $t \rightarrow b \tau \nu$, while the other $t$ decays hadronically. This has however a much softer $\tau$-jet and can be suppressed significantly with the cut (25). Moreover the opposite $\tau$ polarizations for the signal and background can be used to suppress the background further, as discussed earlier. Figure 5 shows the signal and background cross-sections against the fractional $\tau$-jet momentum carried by the charged 
pion (20). The hard charged pion cut of (21) suppresses the background by a factor of 5-6 while retaining almost half the signal cross-section. Moreover the signal $\tau$-jet has a considerably harder $p_{T}$ and larger azimuthal opening angle with the $p_{T}$ in comparison with the background. Consequently the signal has a much broader distribution in the transverse mass of the $\tau$-jet with the $\not p_{T}$, extending upto $M_{H^{ \pm}}$, while the background goes only upto $M_{W}$. Figure 6 shows these distributions both with and without the hard charged pion cut (21). One can effectively separate the $H^{ \pm}$signal from the background and estimate the $H^{ \pm}$ mass from this distribution. The LHC discovery reach of this channel is shown in Fig. 4, which clearly shows it to be the best channel for a heavy $H^{ \pm}$search at large $\tan \beta$. It should be added here that the transition region between $M_{H^{ \pm}}>m_{t}$ and $<m_{t}$ has been recently analysed in [24] by combining the production process of $(22)$ with $(13,14)$. As a result it has been possible to bridge the gap between the two discovery contours of Fig. 4 via the $\tau \nu$ channel.

\subsection{Heavy $H^{ \pm}$Search in the $t b$ Channel}

Let us discuss this first for 3 and then 4 b-tags. In the first case the signal comes from (22), followed by

$$
H^{ \pm} \rightarrow t \bar{b}, \bar{t} b
$$

The background comes from the NLO QCD processes

$$
g g \rightarrow t \bar{t} b \bar{b}, g b \rightarrow t \bar{t} b+h . c ., g g \rightarrow t \bar{t} g
$$

where the gluon jet in the last case can be mistagged as $b$ (with a typical probability of $\sim 1 \%)$. One requires leptonic decay of one of the $t \bar{t}$ pair and hadronic decay of the other with a $p_{T}>30 \mathrm{GeV}$ cut on all the jets [25]. For this cut the $b$-tagging efficiency at LHC is expected to be $\sim 50 \%$. After reconstruction of both the top masses, the remaining (3rd) $b$ quark jet is expected to be hard for the signal $(22,26)$, but soft for the background processes (27). A $p_{T}>80 \mathrm{GeV}$ cut on this $b$-jet improves the signal/background ratio. Finally this $b$-jet is combined with each of the reconstructed top pair to give two entries of $M_{t b}$ per event. For the signal events, one of them corresponds to the $H^{ \pm}$mass while the other constitutes a combinatorial background. Figure 7 shows this invariant mass distribution for the signal along with the above mentioned background processes for different $H^{ \pm}$masses at $\tan \beta=40$ Similar results hold for $\tan \beta \simeq 1.5$. One can check that the significance level of the signal is $S / \sqrt{B} \gtrsim 5[25]$. The corresponding $H^{ \pm}$discovery reaches in the high and low $\tan \beta$ regions are shown in Fig. 4. While the discovery reach via $t b$ is weaker than that via the $\tau \nu$ channel in the high $\tan \beta$ region, the former offers the best $H^{ \pm}$discovery reach in the low $\tan \beta$ region. This is particularly important in view of the fact that the indirect LEP limit shown in Fig. 4 gets significantly weaker with the reported increase in the top quark mass, as discussed earlier. Indeed this $H^{ \pm} \rightarrow t b$ discovery contour constitutes the most robust discovery limit for the MSSM Higgs sector over the low $\tan \beta$ region. On the other hand the $H^{ \pm} \rightarrow \tau \nu$ contour is competitive with that from the $H^{0} / A^{0} \rightarrow \tau \tau$ channel as the best MSSM Higgs discovery limit over the high $\tan \beta$ region. Finally the corresponding $H^{ \pm} \rightarrow \tau \nu$ contour from $t \rightarrow b H^{+}$decay, also shown in Fig. 4, constitutes the best discovery limit of the MSSM Higgs sector over the low $M_{A}$ region (see e.g. Fig. 27 of ref.[1]). 
One can also use $4 b$-tags to look for the $H^{ \pm} \rightarrow t b$ signal [26]. The signal comes from $(23,26)$, and the background from the first process of $(27)$. After the reconstruction of the $t \bar{t}$ pair, both the remaining pair of $b$-jets are expected to be soft for the background, since they come from gluon splitting. For the signal, however, one of them comes from the $H^{ \pm}$decay (26); and hence expected to be hard and uncorrelated with the other $b$-jet. Thus requiring a $p_{T}>120 \mathrm{GeV}$ cut on the harder of the two $b$-jets along with large invariant mass $\left(M_{b b}>120\right.$ $\mathrm{GeV})$ and opening angle $\left(\cos \theta_{b b}<0.75\right)$ for the pair, one can enhance the signal/background ratio substantially. Unfortunately the requirement of $4 b$-tags makes the signal size very small. Moreover the signal contains one soft $b$-jet from (23), for which one has to reduce the $p_{T}$ threshold from 30 to $20 \mathrm{GeV}$. The resulting signal and background cross-sections are shown in Fig. 8 for $\tan \beta=40$. In comparison with Fig. 7 one can see a significant enhancement in the signal/background ratio, but at the cost of a much smaller signal size. Nonetheless this can be used as a supplementary channel for $H^{ \pm}$search, provided one can achieve good $b$-tagging for $p_{T} \sim 20 \mathrm{GeV}$ jets.

\subsection{Heavy $H^{ \pm}$Search in the $W h^{0}$ Channel}

The tree level coupling for this channel is

$$
H^{+} W^{-} h^{0}: \frac{1}{2} g \cos (\beta-\alpha) q_{h}
$$

where $q_{h}$ is the $h^{0}$ momentum in the $H^{+}$rest frame. The LEP limit of $M_{h^{0}} \gtrsim 100 \mathrm{GeV}$ in the MSSM implies that the $H^{ \pm} \rightarrow W h^{0}$ decay channel has at least as high a threshold as the $t b$ channel. The maximum value of its decay BR,

$$
B^{\max }\left(H^{ \pm} \rightarrow W h^{0}\right) \simeq 5 \%,
$$

is reached for $H^{ \pm}$mass near this threshold and low $\tan \beta$. The small BR for this decay channel is due the suppression of the $H^{+} W^{-} h^{0}$ coupling (28) by the $q_{h}$ and the $\cos (\beta-\alpha)$ factors relative to the $H^{+} \bar{t} b$ coupling (10). Note that both the decay channels correspond the same final state, $H^{ \pm} \rightarrow b \bar{b} W$, along with an accompanying top from the production process (22). Nonetheless one can distinguish the $H^{ \pm} \rightarrow W h^{0}$ from the $H^{ \pm} \rightarrow t b$ as well as the corresponding backgrounds (27) by looking for a clustering of the $b \bar{b}$ invariant mass around $M_{h^{0}}$ along with a veto on the second top [27]. Unfortunately the BR of (29) is too small to give a viable signal for this decay channel. Note however that the LEP limit of $M_{h^{0}} \gtrsim 100 \mathrm{GeV}$ does not hold in the CP violating MSSM [5] or the singlet extensions of the MSSM Higgs sector like the NMSSM [6]. Therefore it is possible to have a $W h^{0}$ threshold significantly below $m_{t}$ in these model. Consequently one can have a $H^{ \pm}$boson lighter than the top quark in these models in the low $\tan \beta$ region, which can dominantly decay into the $W h^{0}$ channel. Thus it is possible to have spectacular $t \rightarrow b H^{+} \rightarrow b W h^{0}$ decay signals at LHC in the NMSSM [27] as well as the CP violating MSSM [28].

\section{Concluding Remarks}

Let me conclude by commenting on a few aspects of $H^{ \pm}$boson search, which could not be discussed in this brief review. The associated production of $H^{ \pm}$with $W$ boson has been 
investigated in [29], and the $H^{ \pm} H^{\mp}$ and $H^{ \pm} A^{0}$ productions in [30]. Being second order electroweak processes, however, they give much smaller signals than (22), while suffering from the same background. However one can get potentially large $H^{ \pm}$signal from the decay of strongly produced squarks and gluinos at LHC, which can help to fill in the gap in the intermediate $\tan \beta$ region of Fig. 4 for favourable SUSY parameters [31].

Finally, the virtual SUSY contribution to the NLO correction for $H^{ \pm}$production can be potentially important since it is known to be nondecoupling, i.e. it remains finite even for very large SUSY mass parameters. The reason for this of course is that the $H^{ \pm}$mass is related to the superparticle masses in SUSY models - e.g. in minimal SUGRA model the $H^{ \pm}$mass is of similar size as the sfermion masses. Therefore the two mass scales can not be decoupled. Consequently the calculation of virtual SUSY correction to $H^{ \pm}$production has received a lot of attention [32, 33, 34]. The main contribution comes from the virtual squark-gluino exchange contribution to the $H^{+} \bar{t} b$ vertex. Its effect can be approximated by a renormalisation of the the $m_{b, t}$ factors in the corresponding coupling (10) by $1 /\left(1+\Delta_{b, t}\right)$, where $[1,22]$

$$
\begin{aligned}
\Delta_{b} \simeq & \frac{2 \alpha_{s}}{3 \pi} m_{\tilde{g}}\left(-A_{b}+\mu \tan \beta\right) I\left(m_{\tilde{b} 1}, m_{\tilde{b} 2}, m_{\tilde{g}}\right) \\
I(a, b, c)= & -\frac{a^{2} b^{2} \ln \left(a^{2} / b^{2}\right)+b^{2} c^{2} \ln \left(b^{2} / c^{2}\right)+c^{2} a^{2} \ln \left(c^{2} / a^{2}\right)}{\left(a^{2}-b^{2}\right)\left(b^{2}-c^{2}\right)\left(c^{2}-a^{2}\right)} \\
& \sim 1 / \max \left(a^{2}, b^{2}, c^{2}\right) ;
\end{aligned}
$$

and there is a similar expression for $\Delta_{t}$. Thus in the large $\tan \beta$ region, where the $m_{b}$ term dominates the $H^{+} \bar{t} b$ coupling, and for $m_{\tilde{g}} \gg m_{\tilde{b} 1,2}$, we get

$$
\Delta_{b} \sim \frac{2 \alpha_{s}}{3 \pi} \frac{\mu \tan \beta}{m_{\tilde{g}}},
$$

which can be very large for $|\mu| \gg m_{\tilde{g}}$ [34]. On the other hand in most SUSY models of common interest we have $|\mu| \sim M_{Z}$ for naturalness, while $m_{\tilde{g}} \gg M_{Z}$. Therefore the above SUSY correction has only modest effect on $H^{ \pm}$production in these models. Indeed a systematic study of this effect for the 'snowmass points and slopes [35]', carried out in [22], shows that the SUSY correction to the cross-section for the LO process (22) remains $\lesssim 20 \%$ for $\tan \beta \lesssim 30$. This is true not only for minimal SUGRA but for other popular alternatives like gauge and anomaly mediated SUSY breaking models as well. As mentioned earlier, the theoretical uncertainly in the estimate of the NLO QCD correction ( $K$ factor) in the SM is also $\sim 20 \%$ [22]. Therefore one need not worry too much about the effect of SUSY quantum correction on the $H^{ \pm}$boson signal at LHC.

\section{References}

[1] For a recent review see M. Carena and H. Haber, Prog. Part. Nucl. Phys. 50, 63 (2003) [hep-ph/0208209].

[2] K. Hagiwara et al. (Particle Data Group), Phys. Rev. D66, 010001 (2002) (URL: http://pdg.lbl.gov) 
[3] CDF and D0 Collaborations and Tevatron Electroweak Working Group, hepex/0404010.

[4] G. Degrassi, S. Hainemeyer, W. Hollik, P. Slavich and G. Weiglein, Euro. Phys. J. C28, $133(2003)$.

[5] M. Carena, J. Ellis, S. Mrenna, A. Pilaftsis and C.E.M. Wagner, Nucl. Phys. B659, 145 (2003).

[6] M. Drees, E. Ma, P.N. Pandita, D.P. Roy and S. Vempati, Phys. Lett. B433, 346 (1998); see also C. Panagiotakopoulos and A. Pilftsis, Phys. Lett. B505, 184 (2001).

[7] A. Mendez and A. Pomarol, Phys. Lett. B252, 461 (1990); C.S. Li and R.J. Oakes, Phys. Rev. D43, 855 (1991); M. Drees and D.P. Roy, Phys. Lett. B269, 155 (1991).

[8] S. Moretti and W.J. Stirling, Phys. Lett. B347, 291 (1995); B366, 451 (E) (1996); A. Djouadi, J. Kalinowski and P.M. Zerwas, Z. Phys. C70, 435 (1996).

[9] E. Ma, D.P. Roy and J. Wudka, Phys. Rev. Lett. 80, 1162 (1998).

[10] E. Keith, E. Ma and D.P. Roy, Phys. Rev. D56, R5306 (1997).

[11] S. Katani et al., Phys. Lett. B378, 329 (1996); E.L. Berger and H. Contopanagos, Phys. Rev. D54, 3085 (1996).

[12] Dø Collaboration: Phys. Rev. Lett. 82, 4975 (1999).

[13] CDF Collaboration: Phys. Rev. D62, 012004 (2000).

[14] CDF Collaboration: Phys. Rev. Lett. 79, 357 (1997).

[15] M. Guchait and D.P. Roy, Phys. Rev. D55, 7263 (1997).

[16] CDF Collaboration: Phys. Rev. D62, 012004 (2000).

[17] S. Raychaudhuri and D.P. Roy, Phys. Rev. D52, 1556 (1995); Phys. Rev. D53, 4902 (1996).

[18] B.K. Bullock, H. Hagiwara and A.D. Martin, Nucl. Phys. B395, 499 (1993).

[19] K.A. Assamagan, Y. Coadou and A. Deandrea, Eur. Phys. J C4, 9 (2002); see also D. Denegri et al., CMS Note 2001/032, hep-ph/0112045.

[20] A.C. Bawa, C.S. Kim and A.D. Martin, Z. Phys. C47, 75 (1990); J.F. Gunion, Phys. Lett. B322, 125 (1994); V. Barger, R.J.N. Phillips and D.P. Roy, Phys. Lett. B324, 236 (1994).

[21] S.H. Zhu, Phys. Rev. D67, 075006 (2003).

[22] T. Plehn, Phys. Rev. D67, 014018 (2003); E.L. Berger, T. Han, J. Jiang and T. Plehn, hep-ph/0312286. 
[23] D.P. Roy, Phys. Lett. B459, 607 (1999).

[24] K.A. Assamagan, M. Guchait and S. Moretti, hep-ph/0402057; see also F. Borzumati, J.L. Kneur and N. Polonsky, Phys. Rev. D60, 115011 (1999).

[25] S. Moretti and D.P. Roy, Phys. Lett. B470, 209 (1999).

[26] D.J. Miller, S. Moretti, D.P. Roy and W.J. Stirling, Phys. Rev. D61, 055011 (2000); see also K.A. Assamagan and N. Gollub, hep-ph/0406013.

[27] M. Drees, M. Guchait and D.P. Roy, Phys. Lett. B471, 39 (1999).

[28] D.K. Ghosh, R.M. Godbole and D.P. Roy (in preparation).

[29] A.A. Barrientos Bendezu and B.A. Kniehl, Phys. Rev. D59, 015009 (1999); S. Moretti and K. Odagiri, Phys. Rev. D59, 055008 (1999); O. Brein, H. Hollik and S. Kanemura, Phys. Rev. D63, 095001 (2001).

[30] A. Kraus, T. Plehn, M. Spira and P.M. Zerwas, Nucl. Phys. B519, 85 (1998); O. Brein and H. Hollik, Eur. Phys. J. C13, 175 (2000); A.A. Barrientos Bendezu and B.A. Kniehl, Nucl. Phys. B568, 305 (2000). For associated $H^{ \pm} A^{0}$ production see Q.H. Cao, S. Kanemura and C.P. Yuan, Phys. Rev. D69, 075008 (2004).

[31] M. Bisset, M. Guchait and S. Moretti, Eur. Phys. J. C19, 143 (2001); A. Datta, A. Djouadi, M. Guchait and Y. Mambrini, Phys. Rev. D65, 015007 (2002).

[32] L. Hall, R. Rattazzi and U. Sarid, Phys. Rev. D50, 7048 (1994); M. Carena, M. Olechowski, S. Pokorski and C.E.M. Wagner, Nucl. Phys. B426, 269 (1994).

[33] J.A. Coarasa, R.A. Jimenez and J. Sola, Phys. Lett. B389, 312 (1996); R.A. Jimenez and J. Sola, Phys. Lett. B389, 53 (1996); A. Bartl, H. Eberl, K. Hikasa, K. Kon, W. Majerotto and Y. Yamada, Phys. Lett. B378, 167 (1996).

[34] A. Belyaev, D. Garcia, J. Guasch and J. Sola, Phys. Rev. D65, 031701 (2002); JHEP 0206, 059 (2002).

[35] B.C. Allanach et al., in Proc. of the APS/DPF/DPB Summer Study on the Future of Particle Physics (Snowmass 2001) Eur. Phys. J. C25, 113 (2002). 


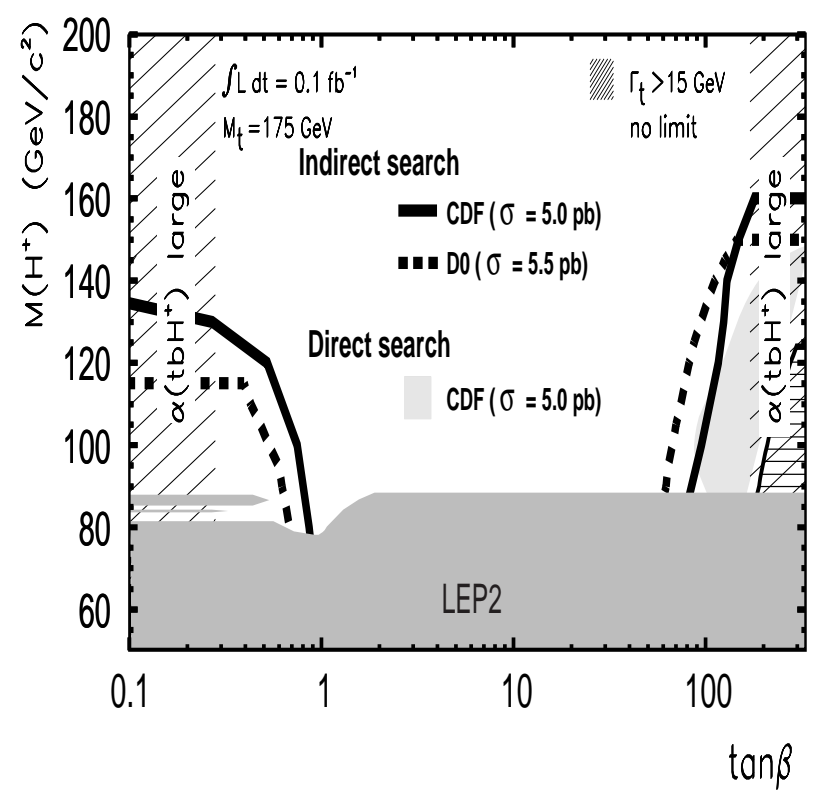

Figure 1: The 95\% CL limits in $M_{H^{ \pm}}-\tan \beta$ plane from LEP-2 (dark grey band) and Tevatron [2]. The Tevatron indirect search limits from $D \emptyset$ [12] and CDF [13] experiments are shown along with the direct search limit from CDF [14]. The cross-hatched regions at extreme values of $\tan \beta$ lie outside the perturbative bounds of eq.(12).

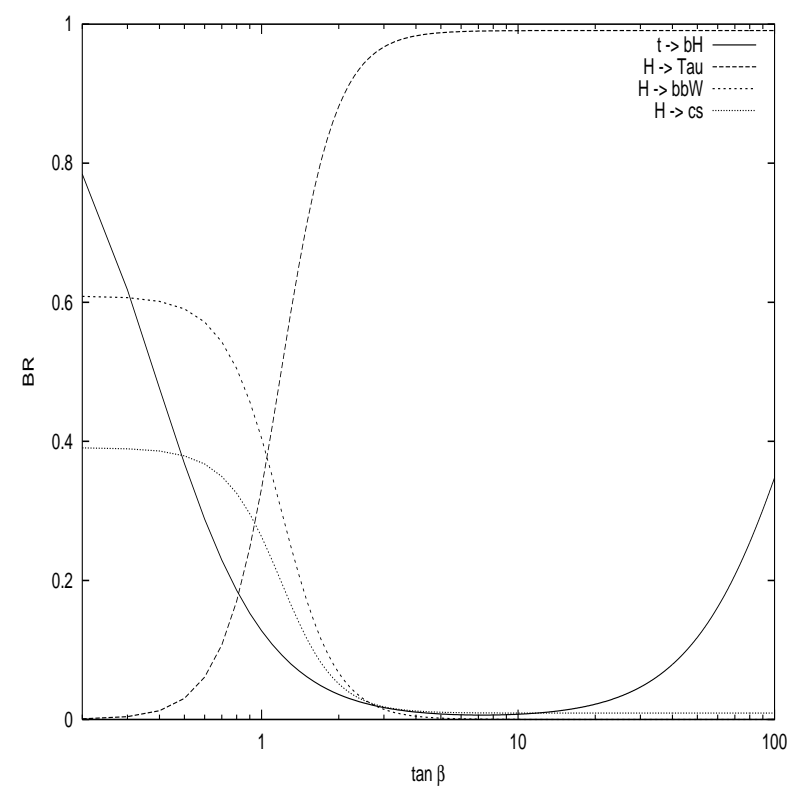

Figure 2: The Branching Ratio of top decay into a $140 \mathrm{GeV} H^{ \pm}$boson (14) shown against $\tan \beta$ along with those for the three main decay modes (15) of this $H^{ \pm}$boson. 


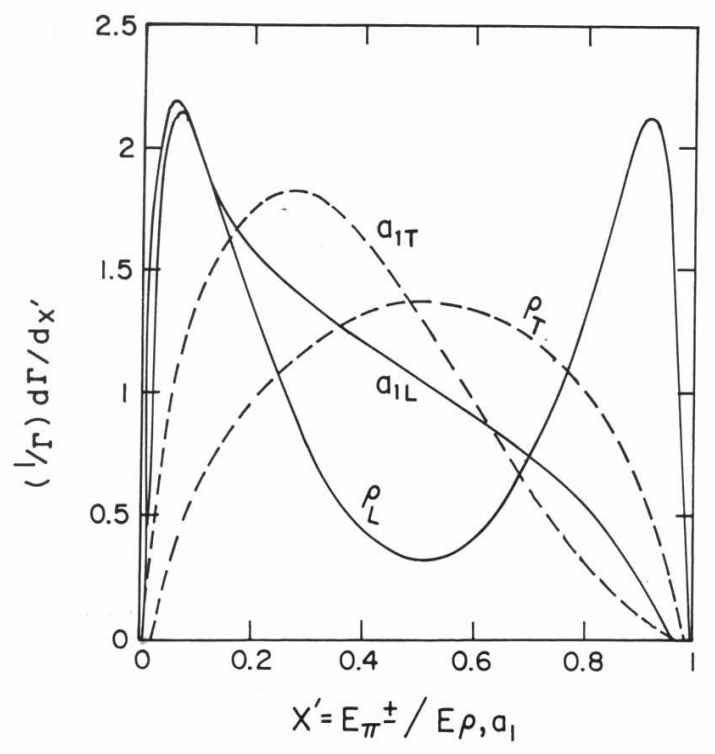

Figure 3: Distributions of the normalised decay widths of $\tau^{ \pm}$via $\rho_{L, T}^{ \pm} \rightarrow \pi^{ \pm} \pi^{0}$ and $a_{1 L, T}^{ \pm} \rightarrow$ $\pi^{ \pm} \pi^{0} \pi^{0}$ in the momentum fraction carried by the charged pion [17]. On this plot the $\tau^{ \pm} \rightarrow$ $\pi^{ \pm} \nu$ decay would correspond to a $\delta$-function at $x^{\prime}=1$.

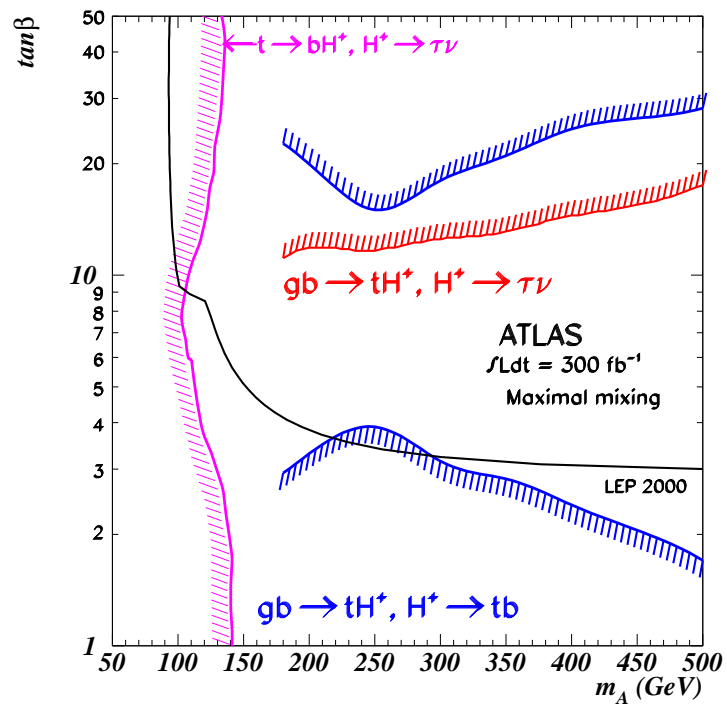

Figure 4: The 5- $\sigma H^{ \pm}$boson discovery contours of the ATLAS experiment at LHS from $t \rightarrow b H^{+}, H^{+} \rightarrow \tau \nu$ (vertical); $g b \rightarrow t H^{-}, H^{-} \tau \nu$ (middle horizontal) and $g b \rightarrow t H^{-}, H^{-} \rightarrow$ $\bar{t} b$ (upper and lower horizontal) channels [19]. One can see similar contours for the CMS experiment in the second paper of ref.[19]. The horizontal part of indirect LEP limit shown here has weakened significantly now as explained in the text. 


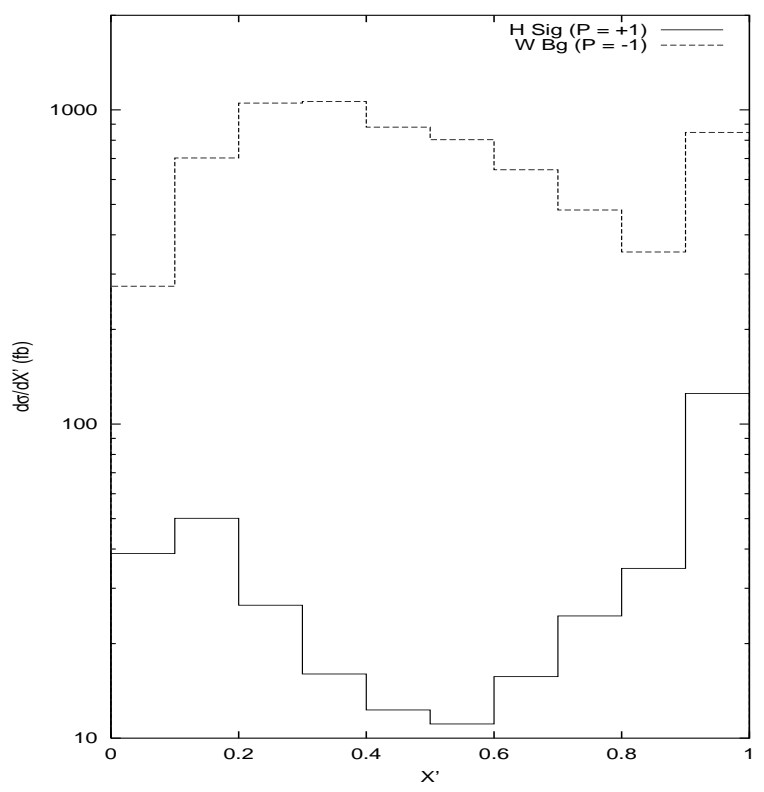

Figure 5: The LHC cross-section for a $300 \mathrm{GeV} H^{ \pm}$signal at $\tan \beta=40$ shown along with the $t \bar{t}$ background in the 1-prong $\tau$-jet channel, as functions of the $\tau$-jet momentum fraction carried by the charged pion.
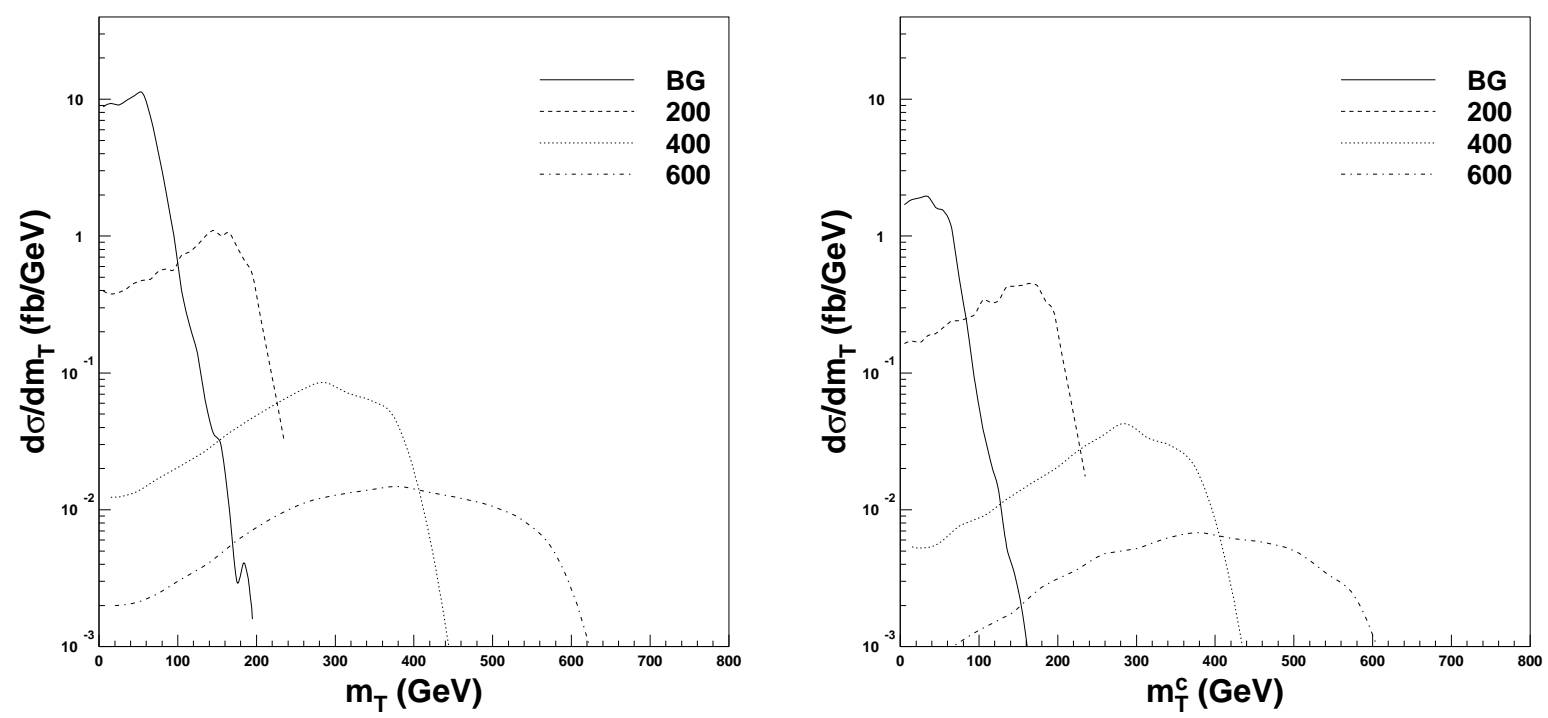

Figure 6: Distributions of the $H^{+}$signal and the $t \bar{t}$ background cross-sections in the transverse mass of the $\tau$-jet with $\not_{T}$ for (left) all 1-prong $\tau$-jets, and (right) those with the charged pion carrying $>80 \%$ of the $\tau$-jet momentum $\left(M_{H^{ \pm}}=200,400,600 \mathrm{GeV}\right.$ and $\left.\tan \beta=40\right)$ [23]. 


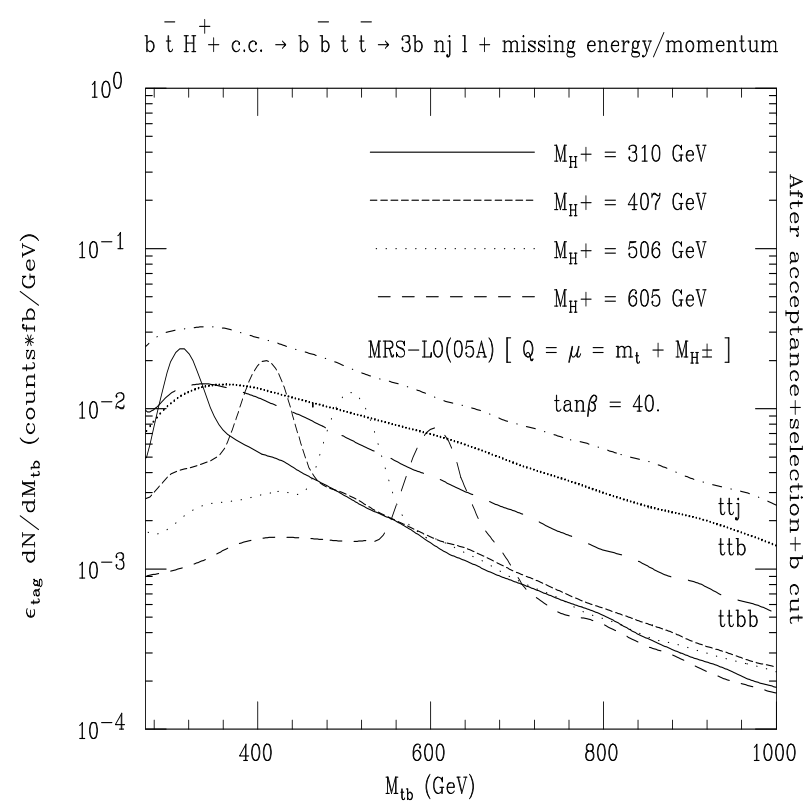

Figure 7: The reconstructed $t b$ invariant mass distribution of the $H^{ \pm}$signal and different QCD backgrounds in the isolated lepton plus multijet channel with $3 b$-tags [25].

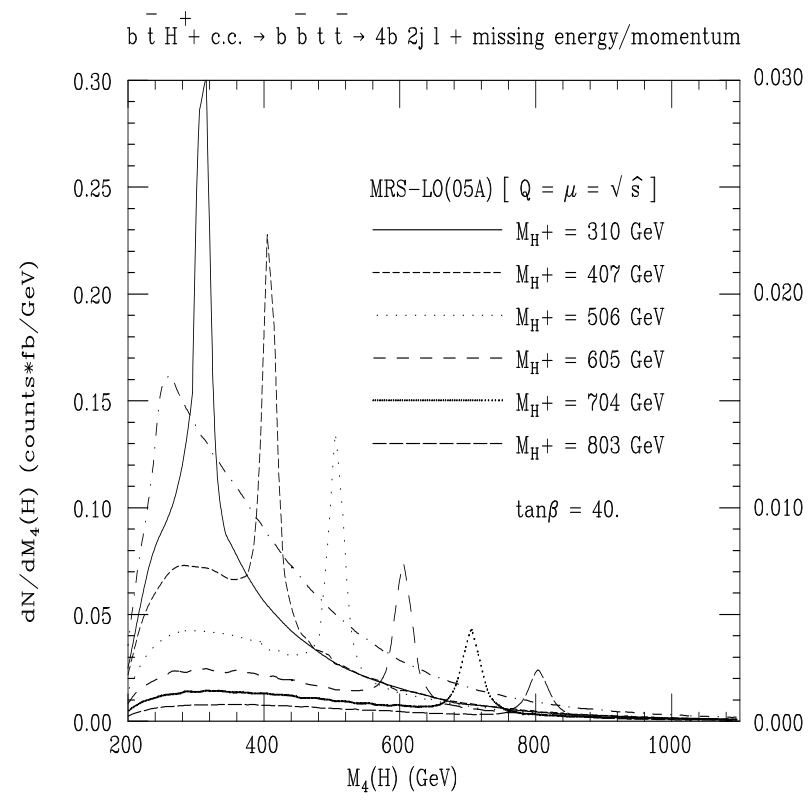

Figure 8: The reconstructed $t b$ invariant mass distribution of the $H^{ \pm}$signal and the QCD background in the isolated lepton plus multijet channel with $4 b$-tags [26]. The scale on the right corresponds to applying a $b$-tagging efficiency factor $\epsilon_{b}^{4}=0.1$. 